\title{
Equilibrium constants of triethanolamine in major seawater salts
}

\author{
I. Brandariz', P. Castro, M. Montes, F. Penedo, M.E. Sastre de Vicente \\ Departamento de Química Física e Ingeniería Química I, Facultad de Ciencias, Campus \\ da Zapateira, c. Alejandro de la Sota 1, E-15008 A Coruña, Spain
}

\section{Marine Chemistry}

Volume 102, Issues 3-4, 5 December 2006, Pages 291-299

Received 26 April 2006, Accepted 30 May 2006, Available online 12 July 2006

doi:10.1016/j.marchem.2006.05.005

\begin{abstract}
Acid-base equilibrium constants of triethanolamine (TEA) have been determined by potentiometric titrations with a glass electrode, at $25^{\circ} \mathrm{C}$. Ionic strength was kept constant with only one electrolyte (using one of these salts: $\mathrm{NaCl}, \mathrm{KCl}, \mathrm{MgCl}_{2}$ or $\mathrm{CaCl}_{2}$ ), with binary mixtures of $\mathrm{MgCl}_{2}$ and $\mathrm{CaCl}_{2}$, and finally, in a solution with a composition approximately similar to that of natural seawater without sulfate. Equilibrium constants have been expressed in function of ionic strength by means of Pitzer equations and interaction parameters proposed in this theory have been obtained. It has been found that acid-base behaviour of TEA depends greatly on the salt used: basicity of TEA is decreased by $\mathrm{CaCl}_{2}$, while it is increased by the other electrolytes used in this work.
\end{abstract}

\section{Keywords}

Pitzer model; Triethanolamine; Potentiometry; Ionic strength; Seawater; Acid-base equilibrium; Stoichiometric constants 


\section{Introduction}

In many cases, the method used by industries to prevent the release of large amounts of $\mathrm{CO}_{2}$ into the atmosphere, involves $\mathrm{CO}_{2}$ removal by chemical absorption/desorption processes with alkanolamine solutions. Triethanolamine (TEA) is one of the most commonly used alkanolamines in these industrial treatments (Horng and Li, 2002). An important factor affecting the $\mathrm{CO}_{2}$ absorption capacity is the basicity of the amine used as absorbent (Benitez-Garcia et al., 1991 and Bonenfant et al., 2003). Therefore, the knowledge of this property in a wide range of conditions is of primary importance for the understanding of the physical chemistry of the absorption process. Besides, TEA is so widely used that it is present in the natural environment (West and Gonsior, 1996), so we have considered interesting to study the acid-base behaviour of TEA in saline media, a subject scarcely treated in literature (Antelo et al., 1984 and Herrero et al., 1991), and the selected salts used in this work are those of seawater. The dependence of acid-base equilibrium constants on salt concentration, even in complex mixtures of electrolytes as seawater, can be described by means of Pitzer model, several examples can be found in the literature (De Stefano et al., 2000, Crea et al., 2006 and Sharma et al., 2006). A brief outline of the model and its application to TEA equilibria is given below.

The protonation equilibrium of triethanolamine can be represented by,

$$
\mathrm{NH}^{+}=\mathrm{N}+\mathrm{H}^{+}
$$

where $\mathrm{N}$ denotes the neutral amine molecule. Protonation constant for this equilibrium can be formulated by,

$$
K^{T}=\frac{[\mathrm{N}]\left[\mathrm{H}^{+}\right]}{\left[\mathrm{NH}^{+}\right]} \frac{\gamma_{\mathrm{N}} \gamma_{\mathrm{H}^{+}}}{\gamma_{\mathrm{NH}^{+}}}=K^{*} \frac{\gamma_{\mathrm{N}} \gamma_{\mathrm{H}^{+}}}{\gamma_{\mathrm{NH}^{+}}}
$$

where $K^{T}$ represents the thermodynamic equilibrium constant, $K^{*}$ the stoichiometric one, and $\gamma i$ is the activity coefficient of the species indicated by the subscript. Taking logarithms one obtains,

$$
-\ln K^{*}=-\ln K^{T}+\ln \gamma_{\mathrm{N}}+\ln \gamma_{\mathrm{H}^{+}}-\ln \gamma_{\mathrm{NH}^{+}}
$$

Activity coefficients of the species appearing in the equilibrium can be expressed by means of Pitzer model. To do so, equations for activity coefficient of neutral molecule, $\ln \gamma{ }_{\mathrm{N}}$, and for a positive ion, $\ln \gamma \mathrm{M}^{+}$(where $\mathrm{M}^{+}$represents $\mathrm{H}^{+}$or $\mathrm{NH}^{+}$), are required. $\ln \gamma_{\mathrm{M}^{+}}{ }^{+}$is expressed by Pitzer as, ( Pitzer, 1991) 


$$
\begin{aligned}
& \ln \gamma_{\mathrm{M}}=z_{\mathrm{M}}^{2}\left[f^{\gamma}+\sum_{\mathrm{c}} \sum_{\mathrm{a}} m_{\mathrm{c}} m_{\mathrm{a}} B_{\mathrm{ca}}^{\prime}+\sum_{\mathrm{c}<} \sum_{\mathrm{c}^{\prime}} m_{\mathrm{c}} m_{\mathrm{c} \prime} \Phi_{\mathrm{cc} \prime}+\sum_{\mathrm{a}<} \sum_{\mathrm{a}} m_{\mathrm{a}} m_{\mathrm{a} \prime} \Phi_{\mathrm{aa} \prime}\right]+ \\
& \sum_{\mathrm{a}} m_{\mathrm{a}}\left(2 B_{\mathrm{Ma}}+Z C_{\mathrm{Ma}}\right)+\sum_{\mathrm{c}} m_{\mathrm{c}}\left(2 \Phi_{\mathrm{Mc}}+\sum_{\mathrm{a}} m_{\mathrm{a}} \Psi_{\mathrm{Mca}}\right)+\sum_{\mathrm{a}<} \sum_{\mathrm{a}}, m_{\mathrm{a}} m_{\mathrm{a} /} \Psi_{\mathrm{Maa} \prime} \\
& +z_{\mathrm{M}} \sum_{\mathrm{c} \cdot} \sum_{\mathrm{a}} m_{\mathrm{c}} m_{\mathrm{a}} C_{\mathrm{ca}}
\end{aligned}
$$

where the sums are over the cations, $\mathrm{c}$, and over the anions, a; $m i$ is the molality of species $i ; f \gamma$ is a extended form of the Debye-Hückel term that takes into account long-range interactions, and it is given, at $25^{\circ} \mathrm{C}$, by:

$$
f^{\gamma}=-0.3915\left[\frac{\sqrt{I}}{1+1.2 \sqrt{I}}+\frac{2}{1.2} \ln (1+1.2 \sqrt{I})\right]
$$

$B$, the second virial coefficient, and its derivative, $B^{\prime}$, are defined in Pitzer theory by:

$$
B_{\mathrm{Ma}}=\beta_{\mathrm{Ma}}^{(0)}+\frac{\beta_{\mathrm{Ma}}^{(1)}}{2 I}\left[1-(1+2 \sqrt{I}) e^{-2 \sqrt{I}}\right]=\beta_{\mathrm{Ma}}^{(0)}+\frac{\beta_{\mathrm{Ma}}^{(1)}}{2 I} g
$$

and

$$
B_{\mathrm{Ma}}^{\prime}=\frac{\beta_{\mathrm{Ma}}^{(1)}}{2 I^{2}}\left[-1+(1+2 I+2 \sqrt{I}) \mathrm{e}^{-2 \sqrt{I}}\right]=\frac{\beta_{\mathrm{Ma}}^{(1)}}{2 I^{2}} g^{\prime}
$$

where we have called $\begin{aligned} & g=1-(1+2 \sqrt{I}) \mathrm{e}^{-2 \sqrt{I}} \text { and } g^{\prime}=-1+(1+2 I+ \\ & 2 \sqrt{I}) \mathrm{e}^{-2 \sqrt{I}}\end{aligned}$ besides $Z=\sum_{i} m_{i}\left|z_{i}\right|, \Phi_{i j}=\theta_{i j}+{ }^{E} \theta_{i j}(I)$ and $\Phi_{i j}{ }^{\prime}={ }^{E} \theta_{i j}{ }^{\prime}(I)$

The interaction parameters $\beta_{\mathrm{Ma}}{ }^{(0)}, \beta_{\mathrm{Ma}}{ }^{(1)}$ are specific to the compound $\mathrm{Ma}$, and they represent the short-range interaction in the presence of the solvent between solute particles $M$ and a. The third virial coefficient, $C_{M a}$, represents triple interactions aaM and MMa. Parameter $\Phi i j$ accounts for interactions between ions of like sign (i and j), it arises only for mixed solutions, $E \theta i j(I)$ and $E \theta i j^{\prime}(I)$ account for electrostatic unsymmetrical mixing effects and depends only on the charges of the ions $i$ and $j$, the total ionic strength and on the solvent (Pitzer, 1991), the other term, $\theta i j$, is considered independent of the ionic strength for any particular $i$ and $j$. The term $\psi_{\mathrm{Mca}}$ is related to the triple interactions of two similarly charged ions ( $\mathrm{M}$ and $\mathrm{c}$ ) with and ion of opposite charge (a). 
On the other hand, the Pitzer equation for the logarithm of the activity coefficient of a neutral species, N, is (Pitzer, 1991 and Millero, 2001)

$$
\begin{aligned}
& \ln \gamma_{\mathrm{N}}=2\left(\sum_{\mathrm{c}} m_{\mathrm{c}} \lambda_{\mathrm{Nc}}+\sum_{\mathrm{a}} m_{\mathrm{a}} \lambda_{\mathrm{Na}}\right)+\sum_{\mathrm{c}} \sum_{\mathrm{a}} m_{\mathrm{c}} m_{\mathrm{a}} \zeta_{\mathrm{caN}}+\sum_{\mathrm{c}<} \sum_{\mathrm{c}}, m_{\mathrm{c}} m_{\mathrm{c} \prime} \eta_{\mathrm{Ncc}}+ \\
& \sum_{\mathrm{a}<} \sum_{\mathrm{a}}{ }^{\prime} m_{\mathrm{a}} m_{\mathrm{a} \prime} \eta_{\mathrm{Naa}}
\end{aligned}
$$

$\lambda i j$ is the second virial coefficient and it represents the short-range interaction in the presence of the solvent between solute particles $i$ and $j$; $\zeta i j k$ and $\eta i j k$ accounts for triple interactions among the three species indicated by the subscript. In last equation, it has been assumed that neutral molecule concentration is low enough so that the terms with $m_{\mathrm{N}}$ can be neglected. On the other hand, triple interaction terms are not required for many systems, but, as Pitzer states, they can be significant in others; in the former case, the equation remain the same as that proposed by Setschenow (Pitzer, 1991 and Long and McDevit, 1952). In figure 1 of Brandariz (2006), it can be observed that the behaviour of $\ln \gamma_{\mathrm{N}}$ (TEA) vs $I$ is linear when $\mathrm{NaCl}, \mathrm{KCl}$ or $\mathrm{MgCl}_{2}$ are used; while this is not the case in $\mathrm{CaCl}_{2}$ medium, where a curvature is clearly appreciated. Because of this, Eq. (5)up to the linear term has been used for the former electrolytes, while third virial coefficient is taken into account for $\mathrm{CaCl}_{2}$. $\mathrm{Ln} \gamma_{\mathrm{N}}$ in $\mathrm{NaCl}$, using Pitzer model, Eq. (5), is given by,

$$
\ln \gamma_{\mathrm{N}}=2 m\left(\lambda_{\mathrm{NNa}}+\lambda_{\mathrm{NCl}}\right)=2 I\left(\lambda_{\mathrm{NNa}}+\lambda_{\mathrm{NCl}}\right)=2 I \lambda_{\mathrm{NaCl}}
$$

the corresponding expression for $\mathrm{KCl}$ is obtained by changing $\mathrm{Na}$ for $\mathrm{K}$, while in $\mathrm{CaCl}_{2}$ medium,

$$
\ln \gamma_{\mathrm{N}}=\frac{2}{3} I\left(\lambda_{\mathrm{NCa}}+2 \lambda_{\mathrm{NCl}}\right)+\frac{2}{9} I^{2} \zeta_{\mathrm{NCaCl}}=\frac{2}{3} I \lambda_{\mathrm{CaCl}}+\frac{2}{9} I^{2} \zeta_{\mathrm{NCaCl}}
$$

this equation is valid for $\mathrm{MgCl}_{2}$ when $\mathrm{Ca}$ is substituted by $\mathrm{Mg}$, and besides $\zeta_{\mathrm{NMgCl}}=0$. The values of the interaction parameters are given in table 3 of Brandariz (2006).

Examples of this behaviour can be found in the literature (Long et al., 1951, Gordon and Thorne, 1967a and Gordon and Thorne, 1967b): third virial coefficient has been been used for the activity coefficient of ammonia in ammonium nitrate (Maeda and Kato, 1995) and ammonium sulfate solutions (Maeda and Iwata, 1997) and for $\mathrm{O}_{2}$ in different salts (Clegg and Whitfield, 1991 and Millero et al., 2002). 


\section{Experimental}

A $40 \mathrm{~mL}$ aqueous aliquot, containing TEA(0.0100 mol $\mathrm{L}^{-1}$ ) (MERCK, p.a.), was titrated with a standard solution of hydrochloric acid (MERCK p.a.). The necessary amount of salt $\left(\mathrm{NaCl}, \mathrm{KCl}, \mathrm{MgCl}_{2}\right.$ or $\mathrm{CaCl}_{2}, \mathrm{MERCK}$, p.a.) to adjust the ionic strength to the desired value was added to both solutions. Titrations were carried out in a dualwall cell that was kept at constant temperature $\left(25^{\circ} \mathrm{C}\right)$ by circulating water from a thermostat. Purified nitrogen was bubbled through the solutions in order to ensure thorough homogenization and $\mathrm{CO}_{2}$ removal. A Crison microBu 2030 automatic burette furnished with $2.5 \mathrm{~mL}$ syringe for dispensing the titrant was used. The burette was controlled via a computer that was used to read the emf values from a Crison micropH 2000 pH-meter, connected to two electrodes: a glass electrode Radiometer pHG211 and a reference electrode Radiometer REF201. At constant ionic strength, emf is related to the proton concentration by (May et al., 1982, Brandariz et al., 1998 and Brandariz et al., 2004):

$$
E=E^{0}+s \log \left[\mathrm{H}^{+}\right]
$$

where $s$ and $E^{0}$ are the slope and the electrode formal potential. $E^{0}$ was obtained from the representation of emf vs $\log \left[\mathrm{H}^{+}\right]$when acid (with salt to adjust ionic strength) was added to a solution of the inert electrolyte. Formal potentials were needed, together with potentiometric titrations data, to determine the acid-base equilibrium constants. This task was performed by using MINIQUAD program ( Vacca and Sabatini, 1985).

The $\mathrm{NaCl}$ and $\mathrm{KCl}$ solutions were made by weight, while stock solutions of known molarity (standardized by density measurements) of $\mathrm{CaCl}_{2}$ and $\mathrm{MgCl}_{2}$ were used to dilute to the desired concentration (Millero et al., 1987) using automatic burettes, in all the cases: when one electrolyte was used, when a mixture of $\mathrm{CaCl}_{2}$ and $\mathrm{MgCl}_{2}$ at constant molality was prepared and when a solution with a composition approximately similar to that of natural seawater without sulfate $\left(m(\mathrm{NaCl})=0.4266, m(\mathrm{KCl})=0.01058, m\left(\mathrm{CaCl}_{2}\right)=0.01077\right.$ and $m\left(\mathrm{MgCl}_{2}\right)=0.05518 \mathrm{~mol} \mathrm{~kg}{ }^{-1}$, see Khoo et al., 1977) was made. Densities were measured with an Anton Paar digital densimeter (model 60/602).

On the other hand, determination of the activity coefficients of neutral molecule was performed using partition coefficients and the procedure described in Brandariz (2006). 


\section{Results and discussion}

Stoichiometric constants of TEA obtained in different media are presented in Table 1 and Table 2, in the molality scale. These constants were originally determined using the molar concentration scale, but in order to apply Pitzer equations, it is necessary to use the molality scale. The relationship between molality, $m i$, and molarity, $c i$, for species $i$ in a solution with a density $\rho$, is expressed by the following equation ( Clegg and Whitfield, 1991):

$$
m_{i}=\frac{c_{i}}{\rho-\sum_{\text {salt }} M_{\text {salt }} c_{\text {salt }}}
$$

where $M_{\text {salt }}$ is the molar mass of the salt. As it can be seen, to perform the conversion between concentration scales, density of solutions is needed. The concentration of the inert electrolyte is much higher than that of the reacting species, so density of solutions has been considered equal to that of solutions containing only the salt, that was taken from Sohnel and Novotny (1985). In the case of the mixture of two $\left(\mathrm{CaCl}_{2}\right.$ and $\left.\mathrm{MgCl}_{2}\right)$ or more salts (ASW) densities were measured experimentally.

Table 1.

Acid-base equilibrium constants of TEA vs ionic strength, in different media, at $25^{\circ} \mathrm{C}$ (see Eqs. (1) and (2))

\begin{tabular}{|c|c|c|c|}
\hline \multicolumn{2}{|c|}{$\mathrm{NaCl}$} & \multicolumn{2}{|l|}{$\mathrm{KCl}$} \\
\hline$I$ & $\mathrm{pK}^{*}$ & $I$ & $\mathrm{pK}^{*}$ \\
\hline 0.10 & 7.793 & 0.10 & 7.769 \\
\hline 0.30 & 7.879 & 0.30 & 7.835 \\
\hline 0.51 & 7.892 & 0.51 & 7.895 \\
\hline 0.71 & 7.942 & 0.72 & 7.923 \\
\hline 1.02 & 8.014 & 03 & 7.983 \\
\hline 1.55 & 8.183 & 1.57 & 8.088 \\
\hline 2.09 & 8.304 & 2.13 & 8.263 \\
\hline \multicolumn{2}{|c|}{$\mathrm{MgCl}_{2}$} & \multicolumn{2}{|c|}{$\mathrm{CaCl}_{2}$} \\
\hline$I$ & $\mathrm{pK}^{*}$ & $I$ & $\mathrm{pK}^{*}$ \\
\hline 0.10 & 7.774 & 0.10 & 7.716 \\
\hline 0.50 & 7.832 & 0.51 & 7.595 \\
\hline 1.01 & 7.910 & 1.02 & 7.557 \\
\hline 1.52 & 7.994 & 1.54 & 7.528 \\
\hline 2.06 & 8.028 & 2.06 & 7.495 \\
\hline
\end{tabular}


Table 2.

Acid-base equilibrium constants of triethanolamine, at $25^{\circ} \mathrm{C}$, determined at constant ionic strength $(I=2.06)$, with varying proportions of $\mathrm{MgCl}_{2}$ and $\mathrm{CaCl}_{2}$

\begin{tabular}{l|l|l|}
$I\left(\mathrm{CaCl}_{2}\right)$ & $I\left(\mathrm{MgCl}_{2}\right)$ & $p K^{*}$ \\
\hline 2.06 & 0.00 & 7.493 \\
\hline 1.54 & 0.51 & 7.546 \\
\hline 1.03 & 1.03 & 7.664 \\
0.51 & 1.54 & 7.826 \\
\hline 0.00 & 2.06 & 8.028 \\
\multicolumn{4}{l}{ Molality scale is used. }
\end{tabular}

In the next two sections we proceed to discuss the results obtained a)when one salt is used and b)when more complex mixtures are employed.

\subsection{Acid-base equilibrium of TEA using a single salt to keep the ionic strength constant}

Eq. (3) can be rewritten as:

$$
p K^{*}=p K^{T}+\frac{1}{\ln 10}\left(\ln \gamma_{\mathrm{N}}+\ln \gamma_{\mathrm{H}^{+}}-\ln \gamma_{\mathrm{NH}^{+}}\right)
$$

Experimental data of $p K^{*}$ vs $I$ in the four electrolytes used in this study are represented in Fig. 1. This picture can be compared to figure 1 of Brandariz (2006), where $\ln \gamma_{\mathrm{N}}$ vs $I$ is plotted for the same salts, both figures are quite similar: $p K^{*}$ or $\ln \gamma_{\mathrm{N}}$ in $\mathrm{NaCl}$ and $\mathrm{KCl}$ have the highest values, that are relatively similar in both salts, the lowest values are found in $\mathrm{CaCl}_{2}$, where a curvature is clearly appreciated, and data for $\mathrm{MgCl}_{2}$ lie in the middle. It seems evident that $p K^{*}$ vs $I$ reflects the behaviour $\ln \gamma_{\mathrm{N}}$ vs $I$, what it is understood in view of Eq.(8). Taking into account that $p K T$ is constant (at a fixed temperature), $p K^{*}$ depends on $\ln \gamma_{\mathrm{N}}$ and on the difference $\ln \gamma_{\mathrm{H}}{ }^{+}-\ln \gamma_{\mathrm{NH}}{ }^{+}$, where some terms will cancel out, as it can be seen in the light of Pitzer equations. Using this model, Eq. (4), without $C$ and $\psi$ parameters, that can be omitted at low ionic strength ( Pitzer, 1991), and considering that concentration of TEA can be neglected compared with salt concentration when $\mathrm{NaCl}$ is used as inert electrolyte, activity coefficient of proton is given by,

$$
\ln \gamma_{\mathrm{H}^{+}}=f^{\gamma}+I^{2} B_{\mathrm{NaCl}}^{\prime}+2 I B_{\mathrm{HCl}}+2 I \theta_{\mathrm{NaH}}
$$

where it has been taken into account that $I=m_{\mathrm{Na}}=m_{\mathrm{Cl}}$. The expression for $\ln \gamma_{\mathrm{NH}}{ }^{+}$is exactly equal to Eq. (9) but replacing $\mathrm{H}^{+}$by $\mathrm{NH}^{+}$, and $\ln \gamma_{\mathrm{N}}$ is given by Eq. (6).

Substituting in Eq. (8) the activity coefficients given by Pitzer model, the result is:

$$
p K^{+}=p K^{T}+\frac{2 I}{\ln 10}\left(\lambda_{\mathrm{NaCl}}+\beta_{\mathrm{HCl}}^{0}+\theta_{\mathrm{NaH}}-\beta_{\mathrm{NH}, \mathrm{Cl}}^{0}-\theta_{\mathrm{Na}, \mathrm{NH}}\right)+\frac{g}{\ln 10}\left(\beta_{\mathrm{HCl}}^{1}-\beta_{\mathrm{NH}, \mathrm{Cl}}^{1}\right)
$$


rearranging, Eq. (10) becomes:

$$
\text { fknownNa }=\frac{-2 I}{\ln 10}\left(\beta_{\mathrm{NH}, \mathrm{Cl}}^{0}-\theta_{\mathrm{NaH}}+\theta_{\mathrm{Na}, \mathrm{NH}}\right)-\frac{g}{\ln 10} \beta_{\mathrm{NH}, \mathrm{Cl}}^{1}
$$

where all terms include in fknownNa are known,

$$
\text { fknownNa }=p K^{+}-p K^{T}-\frac{2 I}{\ln 10}\left(\lambda_{\mathrm{NaCl}}+\beta_{\mathrm{HCl}}^{0}\right)-\frac{g}{\ln 10} \beta_{\mathrm{HCl}}^{1}
$$

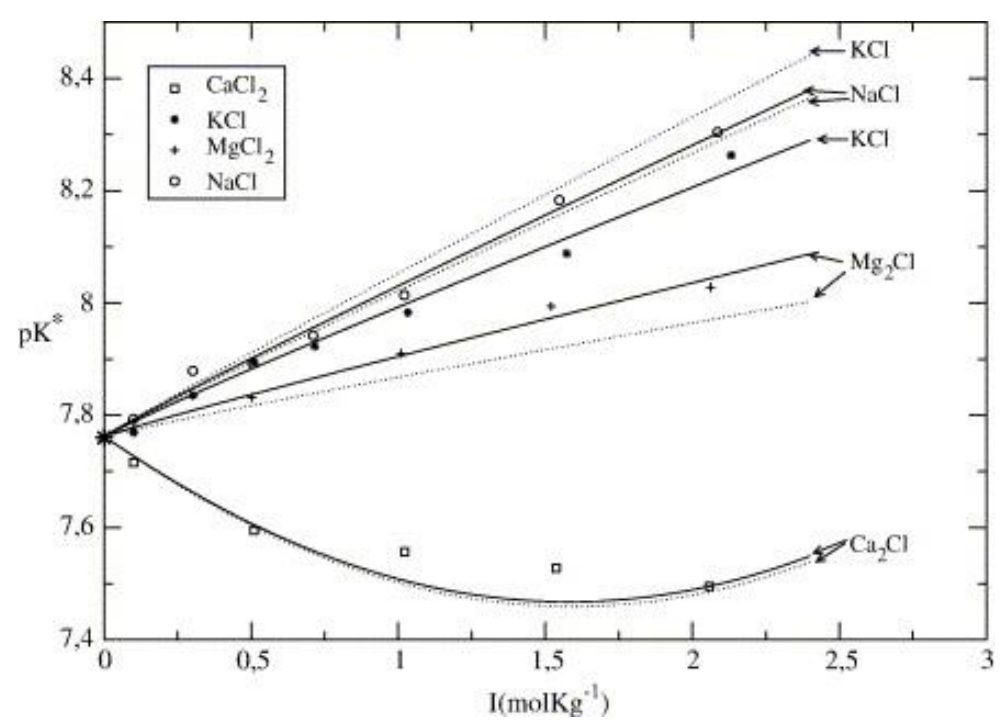

Fig. 1.

$p K^{*}$ vs $I$, symbols represent experimental data, while dotted lines correspond to Pitzer model omitting $\theta$ parameters, and solid lines to Pitzer model with $\theta$ parameters and the same linear term.

All these equations are valid for $\mathrm{KCl}$ when $\mathrm{Na}$ is substituted by $\mathrm{K}$. Proceeding in the same way for $\mathrm{CaCl}_{2}$, the following equation is obtained:

$$
\mathrm{fknownCa}=\frac{-2 I}{\ln 10}\left(\beta_{\mathrm{NH}, \mathrm{Cl}}^{0}-0.5 \theta_{\mathrm{CaH}}+0.5 \theta_{\mathrm{Ca}, \mathrm{NH}}\right)-\frac{g}{\ln 10} \beta_{\mathrm{NH}, \mathrm{Cl}}^{1}
$$

where

$$
\text { fknownCa }=\frac{3}{2}\left[p K^{*}-p K^{T}-\frac{4 I}{3 \ln 10}\left(0.5 \lambda_{\mathrm{CaCl}}+\beta_{\mathrm{HCl}}^{0}\right)-\frac{2 g}{3 \ln 10} \beta_{\mathrm{HCl}}^{1}-\frac{2 I^{2}}{9 \ln 10} \zeta_{\mathrm{NCaCl}}\right]
$$

This equation is valid for $\mathrm{MgCl}_{2}$ when $\mathrm{Ca}$ is substituted by $\mathrm{Mg}$, and remenbering

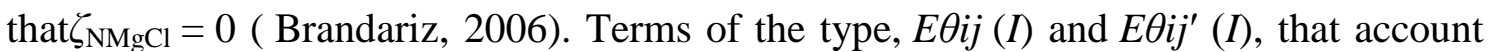
for electrostatic unsymmetrical mixing effects (interactions $\mathrm{Ca}^{+2}, \mathrm{H}^{+}$or $\mathrm{Ca}^{+2}, \mathrm{NH}^{+}$), depends only on the charges of the ions, the total ionic strength and on the solvent, so that they cancel out in Eq. (13). Thermodynamic equilibrium constant, $p K T$, is taken from (Bates and Allen, 1960), $\lambda$ and $\zeta$ from (Brandariz, 2006) and the other parameters appearing in fknown terms are taken from ( Pitzer, 1991), they are gathered in Table 3.

Table 3.

Pitzer parameters, at $25^{\circ} \mathrm{C}$, used through this paper, with the references they have been taken from 


$\begin{array}{ll}\text { Parameter } & \text { Reference } \\ p K T=7.762 & \text { Bates and Allen (1960) } \\ \beta_{\mathrm{HCl}}{ }^{0}=0.1775 \beta_{\mathrm{HCl}}^{1}=0.2945 & \text { Pitzer (1991) } \\ \lambda_{\mathrm{NNa}}+\lambda_{\mathrm{NCl}}=-0.009 \pm 0.005 & \text { Brandariz (2006) } \\ \lambda_{\mathrm{NK}}+\lambda_{\mathrm{NCl}}=0.028 \pm 0.007 & \\ \lambda_{\mathrm{NMg}}+2 \lambda_{\mathrm{NCl}}=-0.250 \pm 0.015 & \\ \lambda_{\mathrm{NCa}}+2 \lambda_{\mathrm{NCl}}=-1.94 \pm 0.09 \zeta_{\mathrm{NCaCl}}=1.3 \pm 0.1 & \end{array}$

The term $\frac{g}{\ln 10} \beta_{\mathrm{NH}, \mathrm{Cl}}^{1}$ is equal for all the electrolytes, as it can be seen in Eqs. (11) and (13), because all of them have the same anion. On the other hand, in the linear term, $\beta_{\mathrm{NH}, \mathrm{Cl}}{ }^{0}$ appears in all the salts, together with $\theta$ parameters, that are different for each electrolyte. Parameter $\theta$ accounts for interactions between ions of like sign ( $i$ and $\mathrm{j}$ ) and since like charged ions repel one another, their short-range interactions are expected to be small ( Pitzer, 1991), therefore, linear terms are expected to be quite similar, with the same value or with small differences because of $\theta$ terms. So as a first approximation, a fit has been performed using all data of the four electrolytes ( Table 1) and considering that the terms that multiply $g$ and $I$ are equal for all the salts, that is supposing that $\theta \approx 0$ in Eqs. (11) and (13). The results obtained are $\beta_{\mathrm{NH}, \mathrm{Cl}}{ }^{0} \approx-0.11$ and $\beta_{\mathrm{NH}, \mathrm{Cl}}{ }^{1} \approx 0.22$, with this parameters and those in Table 3, $p K^{*}$ vs $I$ for Pitzer model have been drawn, using dotted lines, inFig. 1, together with the experimental data. As it can be appreciated, fit is good for sodium and calcium chlorides, but for magnesium salt experimental data lie above the line and for potassium salt they lie below. This problem can be easily solved if $\theta$ terms are taken into account. These parameters can be important to explain experimental data as it can be seen in Kron et al. (1995) and Brandariz et al. (1995), in order to serve as an example, $\ln {\gamma_{\mathrm{H}}}^{+}$vs $I$ for $\mathrm{NaCl}$ has been plotted in Fig. 2, it is evident the change that the inclusion of $\theta$ produces in the curves. Therefore, a new fit has been performed using all data in Table 1, considering that linear terms are different for all the electrolytes, but term in $g$ is the same. The method of dummy variables in multiple regression has been used to achieve this goal (Draper and Smith, 1981), and some subroutines ofPress et al. (1992) were used to perform the linear fit. The parameters obtained are displayed in Table 4, and the curves $p K^{*}$ vs $I$ are plotted for all the salts in Fig. 1 with solid lines. The fit of experimental data is really good, and much better than the first model we tried. 


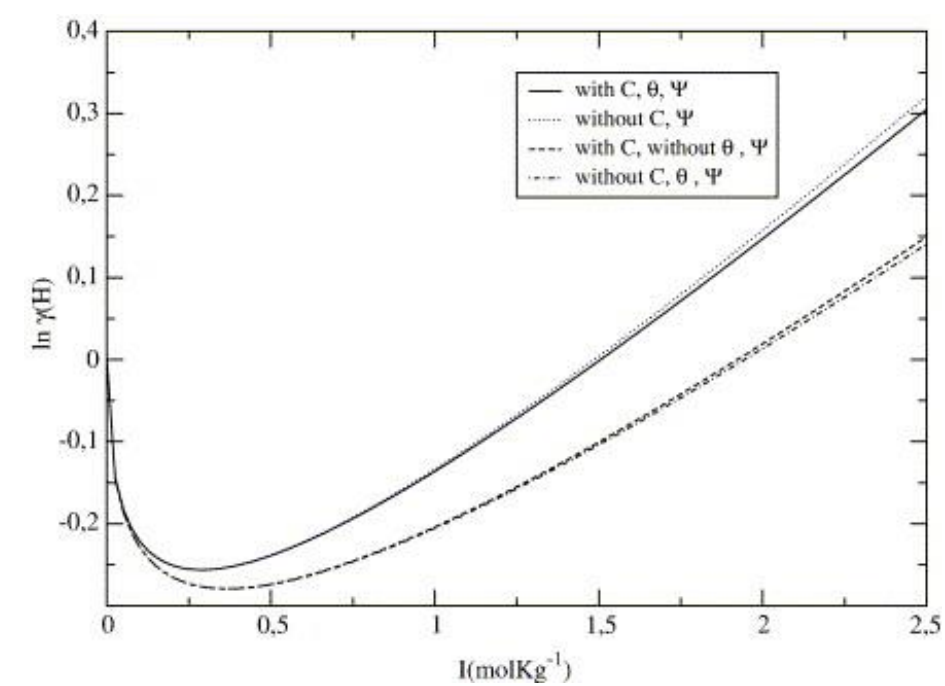

Fig. 2.

$\ln \gamma_{\mathrm{H}+}$ vs $I$ for $\mathrm{NaCl}$ using Pitzer equations, with and without some parameters to emphasize the importance of $\theta$ parameter.

Table 4

Pitzer parameters, at $25^{\circ} \mathrm{C}$

$\beta_{\mathrm{NH}, \mathrm{Cl}}{ }^{1}=0.20 \pm 0.08$

$$
\begin{aligned}
& \beta_{\mathrm{NH}, \mathrm{Cl}}{ }^{0}-\theta_{\mathrm{NaH}}+\theta_{\mathrm{Na}, \mathrm{NH}}=-0.111 \pm 0.021 \\
& \beta_{\mathrm{NH}, \mathrm{Cl}}{ }^{0}-\theta_{\mathrm{KH}}+\theta_{\mathrm{K}, \mathrm{NH}}=-0.032 \pm 0.020 \\
& \beta_{\mathrm{NH}, \mathrm{Cl}}{ }^{0}-0.5 \theta_{\mathrm{MgH}}+0.5 \theta_{\mathrm{Mg}, \mathrm{NH}}=-0.166 \pm 0.020 \\
& \beta_{\mathrm{NH}, \mathrm{Cl}}{ }^{0}-0.5 \theta_{\mathrm{CaH}}+0.5 \theta_{\mathrm{Ca}, \mathrm{NH}}=-0.112 \pm 0.020 \\
& \eta_{\mathrm{NMGCa}}=-2.25( \pm 0.25)
\end{aligned}
$$

\subsection{Acid-base equilibrium of TEA in mixtures of two or more salts}

Acid-base equilibrium constants of triethanolamine, determined at constant ionic strength $(I=2.06)$, with varying proportions of $\mathrm{MgCl}_{2}$ and $\mathrm{CaCl}_{2}$, appear in Table 2 . These experimental data are plotted in Fig. 3, where a curvature is clearly appreciated. Pitzer model, Eq. (4), without C and $\psi$ parameters, was applied successfully to explain experimental data when one single electrolyte was used to keep the ionic strength constant. When a mixture of two electrolytes is employed, in view of Eq. (4), if C and $\psi$ can actually be omitted in the activity coefficient of charged species, the observed curvature in the plot $p K^{*}$ vs I, should be adscribed to the behaviour of $\ln \gamma_{\mathrm{N}}$ vs $I\left(\mathrm{CaCl}_{2}\right)$. In order to verify this hypothesis activity coefficients of the neutral molecule has been determined and are given in Table 5 and plotted in Fig. 4, where a curvature in the experimental data is, again, clearly appreciated; based on this result, one question arises, could be this behaviour explained from that in pure electrolytes? As it is stated in Millero (2001), the behaviour of nonelectrolytes in mixed electrolyte solutions have not been thoroughly studied. The data have been fitted to a quadratic function, that adequately describes the experimental behaviour, as it can be seen in Fig. 4, 


$$
\ln \gamma_{\mathrm{N}}(\operatorname{mixI})=-0.35( \pm 0.01)-1.06( \pm 0.03) I_{\mathrm{Ca}}+0.25( \pm 0.01) I_{\mathrm{Ca}}^{2}
$$

where $I_{\mathrm{Ca}}=3 m_{\mathrm{Ca}}$, being $m_{\mathrm{Ca}}$ the molality of calcium salt. The following combination has been found to be successful in obtaining data in the mixture from that of single electrolytes,

$$
\ln \gamma_{\mathrm{N}}(\operatorname{mix})=\ln \gamma_{\mathrm{N}}\left(\mathrm{MgCl}_{2}\right)+\ln \gamma_{\mathrm{N}}\left(\mathrm{CaCl}_{2}\right)
$$

where $\gamma_{\mathrm{N}}\left(\mathrm{MgCl}_{2}\right)$ and $\gamma_{\mathrm{N}}\left(\mathrm{CaCl}_{2}\right)$ are the activity coefficients of TEA at the same concentration of the salt in the mixture, therefore, using Eq. (7) and data in Table 3, Eq. (16) yields

$$
\ln \gamma_{\mathrm{N}}(\operatorname{mixII})=-0.34( \pm 0.02)-1.12( \pm 0.07) I_{\mathrm{Ca}}+0.28( \pm 0.02) I_{\mathrm{Ca}}^{2}
$$

Eq. (17) can be considered equal to (15) within the experimental error, and both of them seem to be perfectly able to reproduce the experimental data (Fig. 4). On the other hand, if Pitzer model is invoked to explain the dependence $\ln \gamma_{\mathrm{N}}(\mathrm{mix})$ vs $I, \eta_{\mathrm{NMgCa}}$ parameter is needed, using Eq. (5) for the mixture, the result is,

$$
\ln \gamma_{\mathrm{N}}(\operatorname{mix})=2 m_{\mathrm{Ca}} \lambda_{\mathrm{CaCl}}+2 m_{\mathrm{Mg}} \lambda_{\mathrm{MgCl}}+m_{\mathrm{Cl}} m_{\mathrm{Ca}} \zeta_{\mathrm{NCaCl}}+m_{\mathrm{Ca}} m_{\mathrm{Mg}} \eta_{\mathrm{NCaMg}}
$$

if the following expressions are taken into account,

$$
m_{\mathrm{Mg}}=\frac{I}{3}-m_{\mathrm{Ca}}, m_{\mathrm{Cl}}=\frac{2}{3} I, I_{\mathrm{Ca}}=3 m_{\mathrm{Ca}}, I=3 m_{\mathrm{Ca}}+3 m_{\mathrm{Mg}}=I_{\mathrm{Ca}}+I_{\mathrm{Mg}}=2.06,
$$

then Eq. (18) yields,

$$
\begin{aligned}
& \ln \gamma_{\mathrm{N}}(\operatorname{mix})=\frac{2}{3} 2.06 \lambda_{\mathrm{MgCl}}+\frac{2}{3}\left(\lambda_{\mathrm{CaCl}}-\lambda_{\mathrm{MgCl}}+\frac{2.06}{3} \zeta_{\mathrm{NCaCl}}+\frac{2.06}{6} \eta_{\mathrm{NMgCa}}\right) I_{\mathrm{Ca}} \\
& -\frac{1}{9} \eta_{\mathrm{NMgCa}} I_{\mathrm{Ca}}^{2}
\end{aligned}
$$

Comparing (15) and (19)

$$
\frac{-1}{9} \eta_{\mathrm{NMgCa}}=0.25( \pm 0.01) \rightarrow \eta_{\mathrm{NMgCa}}=-2.25( \pm 0.25)
$$

using this value, the other terms in Eq. (19) should be: 


$$
\begin{array}{rl}
\frac{2}{3} 2 & .06 \lambda_{\mathrm{MgCl}}=\frac{2}{3} 2.06(-0.25)=-0.34 \\
& \frac{2}{3}\left(\lambda_{\mathrm{CaCl}}-\lambda_{\mathrm{MgCl}}+\frac{2.06}{3} \zeta_{\mathrm{NaCl}}+\frac{2.06}{6} \eta_{\mathrm{NMgCa}}\right) \\
= & \frac{2}{3}\left[-1.94-(-0.25)+\frac{2.06}{3} 1.3+\frac{2.06}{6}(-2.25)\right] \\
& =-1.05
\end{array}
$$

these values agree very well with those in Eq. (15), therefore it can be stated that, this equation represents the experimental behaviour of Pitzer model with $\eta_{\mathrm{NMgCa}}$. In accordance with this theory, when parameters for the system of neutral solute in a single electrolyte solution are the only ones used, last term, in $I_{\mathrm{Ca}}{ }^{2}$, disappears, $\left(\eta_{\mathrm{NMgCa}}\right.$ is omitted) and the behaviour $\ln \gamma_{\mathrm{N}}$ (mix) vs $I$ would be linear, what is not the case here. It is clear that a quadratic function on $I$ is needed to deal with the activity coefficient of amine neutral molecule in the mixtures. Therefore, to find $p K^{*}$ (mix) vs I, any of the Eq. (15) or (17) for $\ln \gamma_{\mathrm{N}}$ can be used, in junction with $\left(\ln \gamma_{\mathrm{H}}{ }^{+}-\ln \gamma_{\mathrm{NH}}{ }^{+}\right)$obtained from Pitzer (with Eq. (4) and data in Table 3 and Table 4), the result is,

$$
p K^{*}(\operatorname{mixI})=8.04( \pm 0.05)-0.49( \pm 0.04) I_{\mathrm{Ca}}-0.110( \pm 0.004) I_{\mathrm{C}}^{2}
$$

using Eq. (15), and

$$
p K^{*}(\operatorname{mix} \mathrm{II})=8.04( \pm 0.05)-0.52( \pm 0.05) I_{\mathrm{Ca}}-0.12( \pm 0.01) I_{\mathrm{Ca}}^{2}
$$

using Eq. (17). The curves for both models appear in Fig. 3 together with the quadratic function obtained from the fit of the experimental data $p K^{*}$ (mix) vs I, given by

$$
\begin{aligned}
p K^{*}(\operatorname{mix})= & 8.030( \pm 0.005)-0.45( \pm 0.01) I_{\mathrm{Ca}}-0.092 \\
& \times( \pm 0.0006) I_{\mathrm{Ca}}^{2}
\end{aligned}
$$

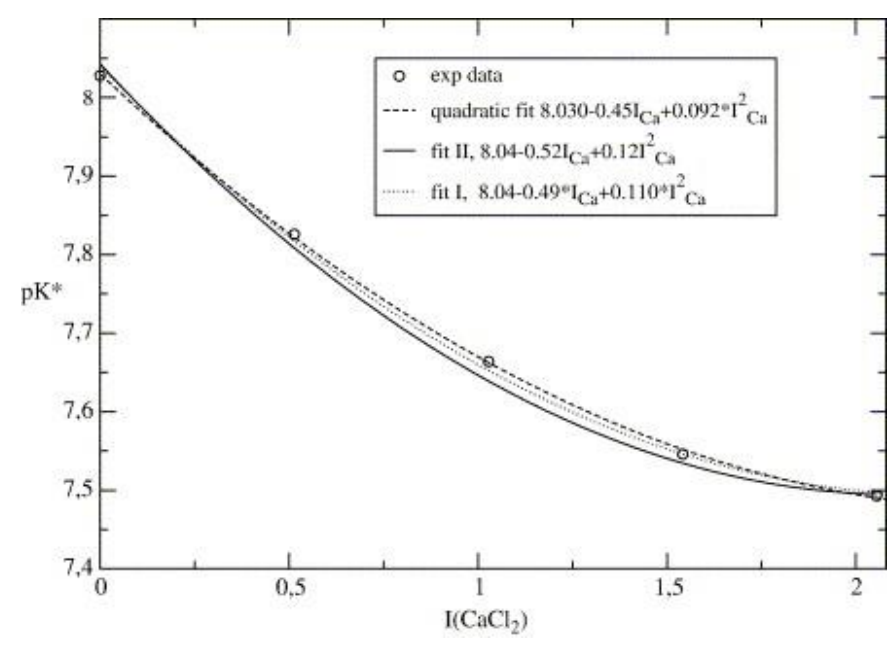

Fig. 3.

$p K^{*}$ vs $I\left(\mathrm{CaCl}_{2}\right)$ in mixtures of $\mathrm{MgCl}_{2}$ and $\mathrm{CaCl}_{2}$, at constant ionic strength $I=2.06$ and at $25^{\circ} \mathrm{C}$, symbols represent experimental data ( Table 2), fit I uses experimental quadratic function for $\ln \gamma_{\mathrm{H}}{ }^{+}$in the mixture, see Eqs. (15) and (20), and fit II uses only data from single electrolytes, see Eqs. (17) and (21). 
Table 5.

Activity coefficient of TEA at $25^{\circ} \mathrm{C}$, determined at constant ionic strength $(I=2.06)$, with varying proportions of $\mathrm{MgCl}_{2}$ and $\mathrm{CaCl}_{2}$

\begin{tabular}{|l|l|l|}
\hline$I\left(\mathrm{CaCl}_{2}\right)$ & $I\left(\mathrm{MgCl}_{2}\right)$ & $\ln \gamma_{\mathrm{N}}$ \\
\hline 2.06 & 0.00 & $-1.46^{\mathrm{a}}$ \\
\hline 1.54 & 0.51 & -1.38 \\
\hline 1.03 & 1.03 & -1.16 \\
\hline 0.51 & 1.54 & -0.84 \\
\hline 0.00 & 2.06 & $-0.34^{\mathrm{a}}$
\end{tabular}

Molality scale is used.

a Interpolated from data of pure electrolyte (Brandariz, 2006).

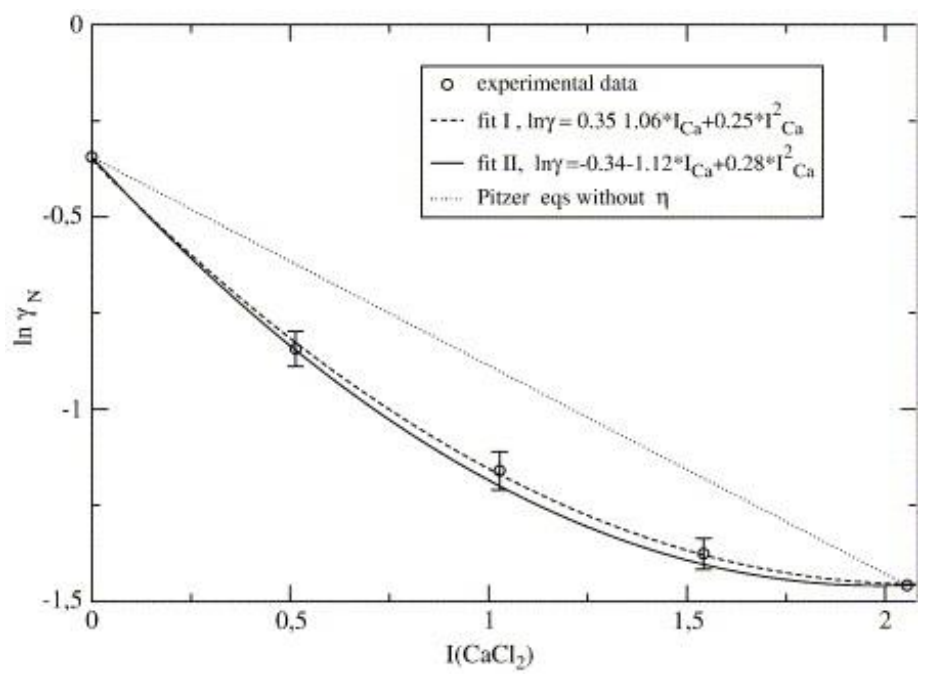

Fig. 4.

$\ln \gamma_{\mathrm{N}}$ vs $I\left(\mathrm{CaCl}_{2}\right)$ in mixtures of $\mathrm{MgCl}_{2}$ and $\mathrm{CaCl}_{2}$, at constant ionic strength $I=2.06$ and $25{ }^{\circ} \mathrm{C}$. Symbols represent experimental data ( Table 5), fit I is a quadratic fit of experimental data, see Eq. (15), and fit II uses only data from single electrolytes, see Eq.(17).

Comparing $p K^{*}$ (mix) with $p K^{*}$ (mixI) and $p K^{*}$ (mixII), or watching Fig. 3 it can be seen that $p K^{*}$ (mixI) is practically equal to $p K^{*}$ (mix) and is slightly better than $p K^{*}$ (mixII), this is perfectly reasonable because this last equation employs all parameters from single electrolytes, actually, if this fact is taken into account, the model can be considered quite good. It is interesting to note that, as it was stated for the single salt solutions, $p K^{*}$ (mix) vs $I$ reflects the behaviour $\ln \gamma_{\mathrm{N}}$ (mix) vs $I$, (see Fig. 3 and Fig. 4). Besides the mixture of two electrolytes, a more complicated system has also been studied in this paper: an approximation to the composition of natural seawater, (without sulfate) found in $\mathrm{Khoo}$

et

al. $(1977): m(\mathrm{NaCl})=0.4266, m(\mathrm{KCl})=0.01058, m\left(\mathrm{CaCl}_{2}\right)=0.01077$ and $m\left(\mathrm{MgCl}_{2}\right)=0.05518 \mathrm{~mol} \mathrm{~kg}^{-1}$. In this medium, the value for the stoichiometric equilibrium constant is, in the molal scale:

$$
p K^{+}(\mathrm{ASW})=7.87, \text { experimental data }
$$


where ASW denotes artificial sea water. The same procedure used in the binary mixtures can be employed to make a theoretical estimation of $p K^{*}(\mathrm{ASW})$, therefore with $\ln \gamma_{\mathrm{N}}$ given by,

$$
\begin{aligned}
& \ln \gamma_{\mathrm{N}}(\operatorname{mix})=\ln \gamma_{\mathrm{N}}\left(\mathrm{MgCl}_{2}\right)+\ln \gamma_{\mathrm{N}}\left(\mathrm{CaCl}_{2}\right)+\ln \gamma_{\mathrm{N}}(\mathrm{NaCl})+\ln \gamma_{\mathrm{N}}(\mathrm{NaCl}) \\
& +\ln \gamma_{\mathrm{N}}(\mathrm{KCl})
\end{aligned}
$$

where $\ln \gamma_{\mathrm{N}}(\mathrm{MCl})$ is the activity coefficient of TEA in a solution with the same concentration of $\mathrm{MCl}$ in the mixture; and with $\left(\ln \gamma_{\mathrm{H}}{ }^{+}-\ln \gamma_{\mathrm{NH}}{ }^{+}\right)$obtained from Pitzer (see Eq. (4) and data in Table 3 and Table 4), it can be obtained,

$$
p K^{*}(\mathrm{ASW})=7.89, \text { estimated with Eq. (23) }
$$

If we would not have performed any work with binary mixtures and we would not realize that $\ln \gamma_{N}$ can be expressed by means of Eq. (23), in that case $p K^{*}$ (ASW) would have been calculated using Pitzer model without $\eta_{\mathrm{NMgCa}}$, and the result would be,

$$
p K^{*}(\mathrm{ASW})=7.90 \text {, estimated by Pitzer model without } \eta_{\mathrm{NMgC}}
$$

both estimations are almost the same because concentration of calcium salt is low in the ASW. The same is true for activity coefficient of TEA, $\ln \gamma_{\mathrm{N}}=-0.077$ is found using Eq. (23) and $\ln \gamma_{\mathrm{N}}=-0.069$, using Pitzer model without $\eta_{\mathrm{NMgCa}}$. This last value was calculated in Brandariz (2006), when data for mixtures were not available yet. The expected distribution coefficients would be ${K_{\mathrm{D}}}^{\mathrm{c}}=0.251$ and ${K_{\mathrm{D}}}^{\mathrm{c}}=0.253$ while the value obtained experimentally in Brandariz (2006) was $K_{\mathrm{D}} \mathrm{c}=$ experimental $=0.261$, as it can be seen, all of them are quite similar.

\section{References}

1. Antelo et al., 1984

J.M. Antelo, F. Arce, J. Casado, M. Sastre, A. Varela

Protonation constants of mono $\mathrm{Ci}^{-}$, and triethanolamine. Influence of the ionic composition of the medium

J. Chem. Eng. Data, 29 (1984), pp. 10-11

2. Bates and Allen, 1960

R.G. Bates, G.F. Allen

Acid dissociation constants and related thermodynamic quantities for triethanolammonium ion in water from 0 to $50{ }^{\circ} \mathrm{C}$

J. Res. NBS, 64A (4) (1960), pp. 343-346

3. Benitez-Garcia et al., 1991

J. Benitez-Garcia, G. Ruiz-Ibañez, H.A. Al-Ghawas, O.C. Sandall 
On the effect of basicity on the kinetics of $\mathrm{CO}_{2}$ absorption in tertiary-amines

Chem. Eng. Sci., 46 (11) (1991), pp. 2927-2931

4. Bonenfant et al., 2003

D. Bonenfant, M. Mimeault, R. Hausler

Determination of the structural features of distinct amines important for the absorption of $\mathrm{CO}_{2}$ and regeneration in aqueous solution

Ind. Eng. Chem. Res., 42 (2003), pp. 3179-3184

5. Brandariz, 2006

I. Brandariz

Salting coefficient of triethanolamine in major seawater salts

Fluid Phase Equilib., 239 (2006), pp. 12-15

6. Brandariz et al., 1995

I. Brandariz, S. Fiol, M. Sastre de Vicente

Study of the influence of ionic strength on the ionic product of water in saline media at $25{ }^{\circ} \mathrm{C}$ using the Pitzer equations

Ber. Bunsenges. Phys. Chem., 99 (5) (1995), pp. 749-752

7. Brandariz et al., 1998

I. Brandariz, T. Vilariño, P. Alonso, R. Herrero, S. Fiol, M.E. Sastre de Vicente

Effect of ionic strength on the formal potential of the glass electrode in various saline media

Talanta, 46 (1998), pp. 1469-1477

8. Brandariz et al., 2004

I. Brandariz, J.L. Barriada, T. Vilariño, M.E. Sastre de Vicente

Comparision of several calibration procedures for glass electrodes in proton concentration

Monatsh. Chem., 135 (2004), pp. 1475-1488

9. Clegg and Whitfield, 1991

S.L. Clegg, M. Whitfield

Activity Coefficient in Natural Waters

K.S. Pitzer (Ed.), Activity Coefficients in Electrolyte Solutions (2nd Ed.), CRC Press, Boca Raton, Fl (1991), pp. 279-434

10. Crea et al., 2006

F. Crea, A. Giacalone, A. Gianguzza, D. Piazzese, S. Sammartano

Modelling of natural and synthetic polyelectrolyte interactions in natural waters by using SIT. Pitzer and ion pairing approaches

Mar. Chem., 99 (2006), pp. 93-105

11. De Stefano et al., 2000

C. De Stefano, C. Foti, A. Gianguzza, S. Sammartano

The interaction of amino acids with the major constituents of natural waters at different ionic strengths 
Mar. Chem., 72 (2000), pp. 61-76

12. Draper and Smith, 1981

N.R. Draper, H. Smith

Applied Regression Analysis

(2nd Ed.)Wiley \& sons, N.Y. (1981), p. 241

13. Gordon and Thorne, 1967a

J.E. Gordon, R.L. Thorne

Salt Effects on the Activity Coefficient of Naphthalene in Mixed Aqueous Electrolyte Solutions. I. Mixtures of Two Salts

J. Phys. Chem., 71 (13) (1967), pp. 4390-4399

14. Gordon and Thorne, $1967 \mathrm{~b}$

J.E. Gordon, R.L. Thorne

Salt effects on non-electrolytes activity coefficients in mixed aqueous electrolyte solutions-II. Artificial and natural sea waters

Geochim. Cosmochim. Acta, 31 (1967), pp. 2433-2443

15. Herrero et al., 1991

R. Herrero, X.L. Armesto, F. Arce, M.S. De Vicente

The protonation constant of triethanolamine in $\mathrm{KBr}$ and $\mathrm{KNO}_{3}$ solutions at $25^{\circ} \mathrm{C}$

J. Solution Chem., 21 (11) (1991), pp. 1185-1193

16. Horng and $\mathrm{Li}, 2002$

S.Y. Horng, M.H. Li

Kinetics of absorption of carbon dioxide into aqueous solutions of monoethanolamine + triethanolamine

Ind. Eng. Chem. Res., 41 (2002), pp. 257-266

17. Khoo et al., 1977

K.H. Khoo, R.W. Ramette, C.H. Culberson, R.G. Bates

Determination of hydrogen ion concentrations in seawater from 5 to $40{ }^{\circ} \mathrm{C}$ : standard potentials at salinities from 20 to $45 \%$

Anal. Chem., 49 (1) (1977), pp. 29-34

18. Kron et al., 1995

I. Kron, S.L. Marshall, P.M. May, G. Hefter, E. Konigsberger

The ionic product of water in highly concentrated aqueous electrolyte solutions

Monatsh. Chem., 126 (1995), pp. 819-837

19. Long and McDevit, 1952

F.A. Long, W.F. McDevit

Activity coefficients of nonelectrolytes solutes in aqueous salt solutions

Chem. Rev., 51 (1952), pp. 119-169

20. Long et al., 1951

F.A. Long, W.F. McDevit, F.B. Dunkle 
Salt effects on the acid-catalized hydrolysis of $\boldsymbol{\gamma}$-butyrolactone, I

J. Phys. Colloid Chem., 55 (1951), pp. 813-829

21. Maeda and Iwata, 1997

M. Maeda, T. Iwata

Dissociation constants of ammonium ion and activity coefficients of ammonia in aqueous ammonium sulfate solutions

J. Chem. Eng. Data, 42 (1997), pp. 1216-1218

22. Maeda and Kato, 1995

M. Maeda, K. Kato

Dissociation constants of ammonium ion and activity coefficients of ammonia in ammonium nitrate solutions

J. Chem. Eng. Data, 40 (1995), pp. 253-256

23. May et al., 1982

P.M. May, D.R. Williams, P.W. Linder, R.G. Torrington

The use of glass electrodes for the determination of formation constants. 1. A definitive method for calibration

Talanta, 29 (4) (1982), pp. 249-256

24. Millero, 2001

F.J. Millero

The Physical Chemistry of Natural Waters

Wiley-Interscience, NY (2001)

25. Millero et al., 1987

F.J. Millero, J.P. Hershey, M. Fernandez

The $\boldsymbol{p K}^{*}$ of $\mathrm{TRISH}^{+}$in $\mathrm{Na}-\mathrm{K}-\mathrm{Mg}-\mathrm{Ca}-\mathrm{Cl}-\mathrm{SO}_{4}$ brines-pH scales

Geochim. Cosmochim. Acta, 51 (1987), pp. 707-711

26. Millero et al., 2002

F.J. Millero, F. Huang, A.L. Laferiere

The solubility of oxygen in the major sea salts and their mixtures at $25{ }^{\circ} \mathrm{C}$

Geochim. Cosmochim. Acta, 66 (13) (2002), pp. 2349-2359

27. Pitzer, 1991

K.S. Pitzer

Ion Interaction Approach: Theory and Data Correlation

K.S. Pitzer (Ed.), Activity Coefficients in Electrolyte Solutions (2nd Ed.), CRC Press, Boca Raton, Fl (1991), pp. 75-153

28. Press et al., 1992

W.H. Press, S.A. Teukolsky, W.T. Vetterling, B.P. Flannery

Numerical Recipes in Fortran

(2nd Ed.)Cambridge University Press (1992)

29. Sharma et al., 2006 
V.K. Sharma, A. Moulin, F.J. Millero, C. De Stefano

Dissociation constants of protonated cysteine species in seawater media

Mar. Chem., 99 (2006), pp. 52-61

30. Sohnel and Novotny, 1985

O. Sohnel, P. Novotny

Densities of Aqueous Solutions of Inorganic Substances

Physical Sciences Data, vol. 22Elsevier, Amsterdam (1985)

31. Vacca and Sabatini, 1985

A. Vacca, A. Sabatini

MINIQUAD and MIQUV

D.J. Legget (Ed.), Computational Methods for the Determination of Formulation Constants, Plenum, N.Y. (1985)

32. West and Gonsior, 1996

R.J. West, S.J. Gonsior

Biodegradation of triethanolamine

Environ. Toxicol. Chem., 15 (4) (1996), pp. 472-480 